\title{
Relations between Stress and Quality of Life among Women in Late Pregnancy: The Parallel Mediating Role of Depressive Symptoms and Sleep Quality
}

\author{
Han Zhang1, Qi Zhang², Tingting Gao', Yixi Kong ${ }^{3}$, Zeying Qin¹,Yueyang Hü ${ }^{3}$, Ruilin Cao', and Songli Mei ${ }^{3 凶}$ \\ ${ }^{1}$ Department of Social Medicine, School of Public Health, Jilin University, Changchun, China \\ 2Department of Nursing Management, School of Nursing, Jilin University, Changchun, China \\ ${ }^{3}$ Department of Maternal and Child Health, School of Public Health, Jilin University, Changchun, China
}

Objective This study aims to examine the parallel multiple mediators of depressive symptoms and sleep quality in the relations between stress and physical health-related quality of life (PHQOL)/mental health-related quality of life (MHQOL) among the women in late pregnancy.

Methods Of 1120 pregnant women participated in the cross-sectional study which consisted of Perceived Stress Scale, Edinburgh Postnatal Depression Scale, Pittsburgh Sleep Quality Index and MOS 12-item Short Form Health Survey. Parallel multiple mediator models were used to analyze the relations between stress, depressive symptoms, sleep quality and PHQOL/MHQOL.

Results The effect of perceived stress on PHQOL was partially through the indirect path of sleep quality $(\beta=-0.061)$. But in the model for MHQOL, depressive symptoms and sleep quality played parallel mediators, and the indirect path effect of depressive symptoms $(\beta=$ -0.179) was higher than sleep quality $(\beta=-0.029)$.

Conclusion The findings contributed to the understanding about the influential mechanism of stress on PHQOL/MHQOL. And it reminded the importance of sleep quality and depressive symptoms for improving QOL in late pregnancy.

Psychiatry Investig 2019;16(5):363-369

Key Words Depression, Pregnant woman, Quality of life, Sleep quality, Parallel multiple mediation model, Mechanism.

\section{INTRODUCTION}

Pregnancy is an important period for women. Many pregnant women experienced significant changes on psychological health, social intercourse and physiological function during antenatal and postpartum period, which had great influence on their lives. ${ }^{1}$ A research conducted in Australia revealed that women in late pregnancy had poorer sleep quality, worse physical health, ${ }^{2}$ and they had a higher proportion of depression. ${ }^{3}$ Therefore, women in this stage should be paid more attention. Quality of life (QOL) refers to individual perception of well-

\footnotetext{
Received: November 26, 2018 Revised: January 15, 2019 Accepted: February 14, 2019

$\triangle$ Correspondence: Songli Mei, $\mathrm{PhD}$

Department of Maternal and Child Health, School of Public Health, Jilin University, No. 1163 Xinmin Street, Changchun, 130021 China

Tel: +86-431 85619454, Fax: +86-431 85619454, E-mail: meisongli@sina.com (c) This is an Open Access article distributed under the terms of the Creative Commons Attribution Non-Commercial License (https://creativecommons.org/licenses/bync/4.0) which permits unrestricted non-commercial use, distribution, and reproduction in any medium, provided the original work is properly cited.
}

being in different aspects of life, including physical function, mental health and social intercourse. ${ }^{4}$ Physical and psychological changes brought by pregnancy affected their perception of QOL. ${ }^{5}$ Researchers found that women with poor QOL were more likely to report pregnancy complications, ${ }^{6}$ and it predicted preterm birth ${ }^{7}$ and low birth weight. ${ }^{8}$ But studies on QOL focused more on the elderly population rather than pregnant women, and the importance of QOL was not got sufficient attention.

Stress is common feeling for pregnant women. ${ }^{9}$ Psychological stress during pregnancy refers to the level of stress is uncontrolled and doubtful, which is associated with depression and acute anxiety. ${ }^{10}$ The prevalence of maternal stress in England ranged from $33 \%$ to $37 \%$ and $5 \%$ to $7 \%$ in Sweden. ${ }^{11} \mathrm{~A}$ study in American reported that $78 \%$ of pregnant women had low or medium level of stress and $6 \%$ of them had high level of stress. ${ }^{9}$ Nearly $36.1 \%$ of pregnant women in Canada experienced certain level of stress during pregnancy. ${ }^{12}$ The theory of psychological stress suggests that stressful life event has ad- 
verse effect on mental health and physical health, and it is related to social factors. Consistent with this theory, many researchers verified the relations between QOL and stress in previous studies. ${ }^{13,14}$ Other study also found that stress increased risk for hypertension and preeclampsia. ${ }^{15}$

Antenatal depressive symptoms were associated with obstetric outcomes, postpartum depressive symptoms and physical function. ${ }^{16}$ Previous study indicated that stress was a predictor for depression, ${ }^{15}$ and high level of stress was related to severer depressive symptoms. There were also scholars found that depression was negatively related to QOL., ${ }^{3,17}$ Additionally, researchers divided QOL into physical health-related quality of life (PHQOL) and mental health-related quality of life (MHQOL), and found that depression was an important predictor both to PHQOL and MHQOL. ${ }^{17}$ According to the results in previous studies, we found that relations between stress, QOL and depression were obvious. But few studies explored the mediating role of depressive symptoms in the relations between perceived stress and QOL among women in late pregnancy. Whether perceived stress affected QOL through psychological symptoms and its influential mechanism were unclear. Determining the influential mechanism of stress on QOL was benefit to adopt necessary measures to improve their QOL. ${ }^{18}$

Sleep quality was an important problem during pregnant period. Study reported that the prevalence of sleep deficiency in the first trimester ranged from $28 \%$ to $38 \% .{ }^{19}$ Pregnant women with sleep deficiency were at a high risk for psychosocial dysfunction, and psychosocial dysfunction was predictor for adverse pregnancy outcome. ${ }^{19}$ The sleep quality was decreased with pregnancy progressed, and sleep quality in late pregnancy was poorest. ${ }^{20} \mathrm{~A}$ systematic review showed that stress, depression, anxiety and sleep quality were the factors related to QOL. ${ }^{21}$ Some other studies also demonstrated the effect of sleep quality on QOL. ${ }^{22,23}$ A study conducted among pregnant women in late pregnancy reported that night sleep length and subjective sleep quality were associated with MHQOL. ${ }^{23}$ To our knowledge, we did not find any researchers studied the mediating role of sleep quality in the relations between perceived stress and PHQOL/MHQOL.

Although previous researchers studied the effect of stress, depressive symptoms, sleep quality on QOL, the parallel multiple mediators of depressive symptoms and sleep quality in the relations between stress and PHQOL/MHQOL among the women in late pregnancy were unclear. Based on previous theory and empirical research, ${ }^{18,24,25}$ we hypothesized that 1) perceived stress was negatively related to $P H Q O L / M H Q O L ;$ 2) depressive symptoms and sleep quality could parallel mediated the relation between stress and MHQOL, but not parallel mediated the relation between stress and PHQOL.

\section{METHODS}

\section{Participants}

This study was conducted in a hospital of Jilin Province between May and September, 2017. Participants were selected from pregnant women awaiting fetal heart rate monitoring in the ambulant clinic by using convenience sampling method. The inclusion criteria were showed as followed: over twenty years old, third trimester of pregnancy, and have ability to complete this questionnaire. The pregnancy women with twins or multiple births and those have mental illness were excluded. Eligible pregnant women were informed the aim and content of this interview, and they could withdraw from the interview at any time. This cross-sectional study was approved by Ethics Committee of the first author's university. We obtained written informed consent from 1151 pregnant women. However, of 31 questionnaires were excluded due to its unavailability. Finally, 1120 pregnant women were included in this study (2017-03-09).

\section{Measures}

Perceived Stress Scale (PSS-10), consulted based on the theory of psychological stress, ${ }^{26}$ was employed to measure their perceived stress in late pregnancy. Each item was rated by scale of 0 (never) to 4 (very often), total score ranged from 0 to 40. A higher score indicated greater pressure she perceived. Chinese version of PSS-10, translated by Lee and Crockett ${ }^{27}$ was found to have high reliability and internal consistency. ${ }^{28}$

Depressive symptom was measured by Edinburgh Postnatal Depression Scale (EPDS), which was built by Cox et al..$^{29}$ and translated into Chinese by Lee et al..$^{30}$ It consisted of 10 items, total score ranged from 0 to 30 . People who scored higher than 9 were considered to have depressive symptoms. It was originally applied to measure depressive symptom in postnatal pregnant women, later it was demonstrated to be suitable for perinatal women and has high reliability and validity. ${ }^{31}$

Pittsburgh Sleep Quality Index (PSQI) compiled by Dr. Buysse and translated into Chinese version by Liu and Tang. ${ }^{32}$ This scale was used to evaluate participants' sleep quality in recent one month. It was suitable for patients with sleep disorder, and general population as well. It was consisted of 7 domains and their sum score was the total score of sleep quality index. High sleep quality index represents poor sleep quality.

MOS 12-item Short Form Health Survey (SF-12) was employed as a tool to measure the quality of life. ${ }^{33}$ It was the shortened version of MOS 36-item Short Form Health Survey (SF-36), and it could be divided into PHQOL and MHQOL. It also consisted of 8 domains, namely physical functioning $(\mathrm{PF})$, role physical (RP), bodily pain (BP), general health $(\mathrm{GH})$, vitality $(\mathrm{VT})$, social functioning $(\mathrm{SF})$, role emotional 
(RE) and mental health (MH). Score of each domain was calculated according to standard algorithm. Previous study has demonstrated that the SF-12 was suitable and valid for Chinese population. ${ }^{34}$

\section{Data analyses}

All statistics were performed by SPSS version 24.0 (IBM Corp., Armonk, NY, USA) for Windows. Correlation analysis was used to analyze the relations among these variables. Simple linear regression model was used to explore confounding factors. Hierarchical linear regression model was used to analyze the parallel mediators (depressive symptoms and sleep quality) in the relations between perceived stress and PHQOL/ MHQOL. Hayes's PROCESS version 2.16 was used to examine the parallel multiple mediating effect. Of $95 \%$ bias corrected confidence intervals (CI) was calculated based on 5000 bootstrapping samples. ${ }^{35} \mathrm{~A}$ p value $<0.05$ and CI do not include zero were considered statistically significant.

\section{RESULTS}

\section{Descriptive statistics of demographic characters}

Nearly three fifth of participants (58.7\%) were 30 years old or younger and average age of all participants was $30.38 \pm 4.08$. Their educational level was generally high, $59.4 \%$ of them had bachelor degree or above. Currently, 23.2\% of them were unemployed in late pregnancy. In regard to family monthly income, most of them were distributed in 3001 Chinese Yuan (CNY) to $9000 \mathrm{CNY}$ (Table 1).

\section{Preliminary analysis}

The means, standard deviations and correlation coefficients were presented in Table 2. Stress, depressive symptoms and sleep quality were positively correlated with each other. Be-

Table 1. Descriptive statistics of social-demographic characters

\begin{tabular}{|c|c|c|c|c|}
\hline Variable & $\mathrm{N}$ & $\%$ & M & SD \\
\hline Age (years) & & & 30.38 & 4.08 \\
\hline 30 and younger & 652 & 58.7 & & \\
\hline 31 and older & 458 & 41.3 & & \\
\hline \multicolumn{5}{|l|}{ Educational level } \\
\hline Senior school or secondary specialized school and lower & 220 & 19.8 & & \\
\hline Junior college & 231 & 20.8 & & \\
\hline Undergraduate & 501 & 45.0 & & \\
\hline Postgraduate and higher & 160 & 14.4 & & \\
\hline \multicolumn{5}{|l|}{ Employed status } \\
\hline Unemployed & 296 & 26.5 & & \\
\hline Employed & 822 & 73.5 & & \\
\hline \multicolumn{5}{|l|}{ Family monthly income (CNY) } \\
\hline 3000 and lower & 103 & 9.3 & & \\
\hline $3001-6000$ & 427 & 38.6 & & \\
\hline $6001-9000$ & 288 & 26.1 & & \\
\hline 9001 and higher & 287 & 26.0 & & \\
\hline
\end{tabular}

$\mathrm{N}$ : number, M: means, SD: standard deviation, CNY: Chinese Yuan

Table 2. The descriptive analysis and correlations among relevant variables

\begin{tabular}{lccccc}
\hline \multicolumn{1}{c}{ Variables } & 1 & 2 & 3 & 4 \\
\hline Stress & 1 & & & \\
Depressive symptoms & $0.642^{* *}$ & 1 & & & \\
Sleep quality & $0.254^{* *}$ & $0.388^{* *}$ & 1 & 1 & \\
PHQOL & $-0.125^{* *}$ & $-0.124^{* *}$ & $-0.255^{* *}$ & $-0.307^{* *}$ & 0.035 \\
MHQOL & $-0.549^{* *}$ & $-0.547^{* *}$ & $6.49 \pm 3.22$ & $43.34 \pm 8.20$ & $49.38 \pm 8.42$ \\
M SD & $14.23 \pm 5.66$ & $6.97 \pm 4.66$ & & \\
\hline
\end{tabular}

${ }^{* *} \mathrm{p}<0.01$. PHQPL: physical health-related quality of life, MHQPL: mental health-related quality of life, M: means, SD: standard deviation 
Table 3. Parallel multiple mediator models for stress on PHQOL/MHQOL

\begin{tabular}{|c|c|c|c|c|c|c|c|c|c|}
\hline \multirow{2}{*}{ Variables } & \multicolumn{3}{|c|}{ Model $1 \mathrm{M}_{1}$ (DS) } & \multicolumn{3}{|c|}{ Model $2 \mathrm{M}_{2}$ (SQ) } & \multicolumn{3}{|c|}{ Model 3 Y (PHQOL/MHQOL) } \\
\hline & Path & $\beta$ & $\mathrm{t}$ & Path & $\beta$ & $\mathrm{t}$ & Path & $\beta$ & $\mathrm{t}$ \\
\hline $\mathrm{X}$ (Stress) & $a_{1}$ & 0.65 & $26.39^{* * *}$ & $\mathrm{a}_{2}$ & 0.24 & $7.47^{* * *}$ & $c^{\prime}$ & $-0.09 /-0.32$ & $-2.28 * /-9.37 * * *$ \\
\hline $\mathrm{M}_{1}(\mathrm{DS})$ & & & & & & & $\mathrm{b}_{1}$ & $0.01 /-0.28$ & $0.33 /-7.92^{* * *}$ \\
\hline $\mathrm{M}_{2}(\mathrm{SQ})$ & & & & & & & $\mathrm{b}_{2}$ & $-0.24 /-0.11$ & $-6.96^{* * *} /-4.06^{* * *}$ \\
\hline Constant & & & -1.82 & & & $8.31^{* * *}$ & & & $45.91^{* * *} / 61.98^{* * *}$ \\
\hline $\mathrm{R}^{2}$ & & \multicolumn{2}{|c|}{0.413} & \multicolumn{3}{|c|}{0.067} & & \multicolumn{2}{|c|}{$0.075 / 0.357$} \\
\hline $\mathrm{F}$ & & \multicolumn{2}{|c|}{$357.250^{* * *}$} & & \multicolumn{2}{|c|}{$24.189^{* * *}$} & & \multicolumn{2}{|c|}{$20.498^{* * *} / 133.479^{* * *}$} \\
\hline
\end{tabular}

${ }^{*} \mathrm{p}<0.05,{ }^{* * *} \mathrm{p}<0.001$. Model 1 regarded family monthly income as confounding factor, model 2 regarded age and educational level as confounding factors, model 3 (PHQOL) regarded age as confounding factor, and model 3 (MHQOL) regarded monthly income as confounding factors. DS: depressive symptoms, SQ: sleep quality, PHQOL: physical health-related quality of life, MHQPL: mental health-related quality of life

sides, those three variables were negatively correlated with PHQOL and MHQOL.

\section{Parallel multiple mediator models for PHQOL/ MHQOL}

Simple linear regression model was used to primarily explore which social-demographic characters may have effect on the parallel multiple mediator models for PHQOL/MHQOL. Results indicated that the relation between family monthly income and depressive symptoms, the relations between age, educational level and sleep quality, the relation between age and PHQOL, and the relation between monthly income and MHQOL were significant. Therefore, these significant socialdemographic characters were included in the later multiple regression models as confounding factors.

We conducted three models to test the parallel mediators of depressive symptoms and sleep quality in the relations between perceived stress and PHQOL/MHQOL (Table 3). Model 1 considered stress as independent variable, depressive symptoms as dependent variable, revealing that stress was positively associated with depressive symptoms ( $\beta=0.647)$. In model 2, stress still played independent variable, but sleep quality was emerged as dependent variable. Results showed that the regression coefficient was significant $(\beta=0.238)$. In model 3 , we regarded stress as independent variable, depressive symptoms and sleep quality as parallel mediate variables and PHQOL/MHQOL as dependent variables. We found that the relation between sleep quality and PHQOL was significant $(\beta=-0.235, p<0.001)$, and the relation between stress and PHQOL was still significant $(\beta=-0.092, p=-0.023)$. So we considered that sleep quality partially mediated the relationship between perceived stress and PHQOL. When we adding mediators in model of MHQOL, we found that the effect of stress, depressive symptoms, sleep quality on MHQOL were all significant.

Test of the total, direct and indirect effects in parallel mul- tiple mediator models were performed using bias-corrected percentile bootstrap method. Results showed that the indirect role of sleep quality between perceived stress and PHQOL was significant, $\beta=-0.061$, lower level of confidence interval $(\mathrm{LLCI})=-0.127$, upper level of confidence interval $(\mathrm{ULCI})=$ -0.057 . Additionally, the indirect effect of depressive symptoms and sleep quality in the relation between stress and MHQOL were significant, $\beta=-0.179 /-0.029$, LLCI $=-0.337 /-0.067$, ULCI= $-0.191 /-0.022$. The indirect effect of depressive symptoms in the relation between stress and PHQOL explained $47.66 \%$ of total effect, and the indirect effect in the model of MHQOL explained 39.39\% of total effect (Table 4 and Figure 1).

\section{Moderating models for PHQOL/MHQOL}

Considering to the possible moderating role of depressive symptoms and sleep quality in the association between stress and quality of life, we conducted a moderation test to examine if the aforementioned results could be measured by a moderating relationship and to detect if any additional variance we can obtained. Results showed that the moderating role of depressive symptoms and sleep quality in the relations between perceived stress and PHQOL/MHQOL were not significant $(\mathrm{p}>0.05)$.

\section{DISCUSSION}

To our knowledge, this was the first study examining the parallel multiple mediators of depressive symptoms and sleep quality in the relations between perceived stress and PHQOL/ MHQOL in Chinese pregnant women. For women in late pregnancy, the effect of perceived stress on PHQOL was partially through the indirect path of sleep quality. But in the model for MHQOL, depressive symptoms and sleep quality played parallel mediators, and the indirect path effect of depressive symptoms was higher than sleep quality. The results contributed to understanding about the influential mechanism of stress on 
Table 4. Total, direct, indirect effects of parallel multiple mediator models

\begin{tabular}{lcccc}
\hline \multicolumn{1}{c}{ Effect } & $\mathrm{B}$ & $\beta$ & LLCI & ULCI \\
\hline $\mathrm{Y}_{1}$ (PHQOL) & & & & \\
$\quad$ Total effect & -0.210 & -0.128 & -0.302 & -0.118 \\
Direct effect & -0.135 & -0.076 & -0.251 & -0.019 \\
Indirect effect $\left(\mathrm{M}_{1}=\mathrm{DS}\right)$ & 0.013 & 0.009 & -0.064 & 0.094 \\
Indirect effect $\left(\mathrm{M}_{2}=\mathrm{SQ}\right)$ & -0.089 & -0.061 & -0.127 & -0.057 \\
$\mathrm{Y}_{2}$ (MHQOL) & & & & \\
Total effect & -0.774 & -0.528 & -0.854 & -0.694 \\
Direct effect & -0.468 & -0.320 & -0.566 & -0.370 \\
Indirect effect $\left(\mathrm{M}_{1}=\mathrm{DS}\right)$ & -0.264 & -0.179 & -0.337 & -0.191 \\
Indirect effect $\left(\mathrm{M}_{2}=\mathrm{SQ}\right)$ & -0.042 & -0.029 & -0.067 & -0.022 \\
\hline
\end{tabular}

DS: depressive symptoms, SQ: sleep quality, PHQOL: physical health-related quality of life, MHQPL: mental health-related quality of life

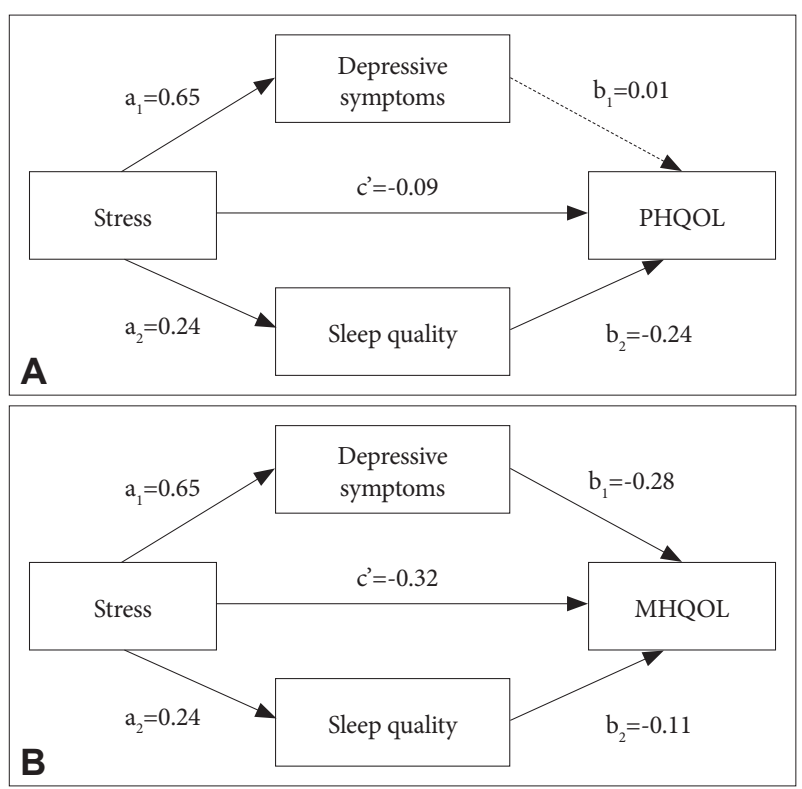

Figure 1. Parallel multiple mediator model. A: PHQOL, B: MHQOL. PHQOL: physical health-related quality of life, MHQPL: mental health-related quality of life.

\section{PHQOL/MHQOL.}

Stress is the commonest emotion during pregnancy, and it has great adverse effect on both the mother and fetus. A massive body of researches documented the predictive function of stress on depressive symptoms, sleep, ${ }^{9,36,37}$ and its negative relationship with QOL. ${ }^{13,14}$ We got similar results in this study. Perceived stress brought by pregnancy had adverse effect on their depressive symptoms, sleep quality, PHQOL and MHQOL. When we adding parallel mediators (depressive symptoms and sleep quality) in the model of PHQOL, the direct of relation between stress and PHQOL was weakened, and stress affected PHQOL partially through the mediating role of sleep quality. A study conducted in Taiwan, China reported a prevalence of sleep disorder at $65.5 \%$ among pregnant women. ${ }^{20}$ Thus the sleep quality of pregnant women should be mentioned in prenatal nursing, especially for the women in late pregnancy. To improve PHQOL, improving sleep quality and reducing sleep disorder were deserved attention.

In the model of parallel multiple mediators for MHQOL, perceived stress was directly related to MHQOL, and depressive symptoms and sleep quality played mediating roles in this relation as well. A study using samples of adolescent pregnancy concluded that negative perception of pregnancy predicted a lower quality of life during pregnancy by increasing the severity of depressive symptoms, ${ }^{24}$ which supported our results of the mediating role of depressive symptoms. In regard to sleep quality, results showed that sleep quality was associated with PHQOL and MHQOL. This finding was similar with previous study. ${ }^{22}$ There were researchers examined the mediating role of sleep in the relation between stress and fat/ vegetables/fruits intake, finding its mediating effect was significant. ${ }^{37}$ Although its dependent variable was not the same as MHQOL, it provided reference for our study. Our results also showed that depressive symptom was more important than sleep quality in the relation between perceived stress and MHQOL, and it had more contribution for this relationship. Depressive symptoms had effect on sleep quality, and people with depressive symptoms were more likely to have sleep disorder. ${ }^{20}$ It was unrealistic to adopt simple measures to improve sleep quality for those pregnant women who had depressive symptoms. To improve sleep quality for pregnant women, relief of their perceived stress and prevention of depressive symptoms were more workable. Thus, we considered that continuous monitoring depressive symptoms during pregnant period had more priority in improving MHQOL.

Quality of life was a comprehensive concept, which was used to describe general health. Earlier study stated that quality of life in the third trimester could predict premature birth. ${ }^{7}$ We have known that there was great difference between PHQOL and MHQOL on related factors. ${ }^{17}$ So we divided QOL into PHQOL and MHQOL, and analyzed its influential mechanism respectively. Findings showed great disparity. Besides, what can be captured from this study was that MHQOL in late pregnancy was better than PHQOL, which is congruent with the study conducted in Macao, China. ${ }^{38}$ Other study also stated that physical health performed worst in late pregnancy, but mental health in postpartum period performed the worst. ${ }^{3}$ Pregnant brought great impairment on their physical function, especially in late pregnancy. But with delivery, their somatic disorders can be alleviated. However, their mental health would be worsened due to problems brought by breast feeding and caregivers. This finding reminded us that it was nec- 
essary to adopt effective measures to decrease sleep disorder and improve PHQOL in late pregnancy.

There were several limitations in this study we should mention. First, these models were based on cross-sectional study rather than a longitudinal study, so we could not draw casual inference. Second, this study was carried out only in one hospital by convenience sampling method, thus it was difficult to generalize the results to other areas due to its limitations. Third, this questionnaire was not designed only for this study, and this study was a part of the project. We did not measure personality in this questionnaire which may affect their perceived stress. These problems should be solved in further research.

In conclusion, the current study revealed the importance of sleep quality and depressive symptoms for improving QOL in late pregnancy. The parallel multiple mediator models in this study contributed to take valid prevention and intervention. First, it is necessary for nurses to strengthen psychological counseling for pregnant women and help them to develop positive attitudes toward pregnancy, thus decrease their stress level and depressive symptoms and boost their QOL. Second, prevention and continuous monitor of depressive symptoms was important in improving MHQOL. Third, nurses in hospital should adopt effective intervention measures to improve sleep quality of pregnant women, and then improve their PHQOL and MHQOL.

\section{Conflicts of Interest}

The authors have no potential conflicts of interest to disclose.

\section{Author Contributions}

$\mathrm{ZH}, \mathrm{ZQ}$ and MS conceived and designed the work, and drafted the manuscript. GT and KY played an important role in interpreting the results and revised the manuscript. QZ, HY and CR acquired data, analyzed the data and revised the manuscript. All authors approved the final version.

\section{ORCID iDs}

\section{Songli Mei}

Han Zhang

https://orcid.org/0000-0003-0507-6373

https://orcid.org/0000-0001-8132-2949

\section{REFERENCES}

1. Faisal-Cury A, Menezes PR. Prevalence of anxiety and depression during pregnancy in a private setting sample. Arch Womens Ment Health 2007;10:25-32.

2. Gartland D, Brown SS, Perlen S. Women's health in early pregnancy: findings from an Australian nulliparous cohort study. Aust N Z J Obstet Gynaecol 2010;50:413-418.

3. Setse R, Grogan R, Pham L, Cooper LA, Strobino D, Powe NR, et al. Longitudinal study of depressive symptoms and health-related quality of life during pregnancy and after delivery: the Health Status in Pregnancy (HIP) study. Matern Child Health J 2009;13:577-587.

4. Buratta V, Frova L, Gargiulo L, Gianicolo E, Prati S, Quattrociocchi L. Copenhagen: EUROHIS - Developing Common Instruments for Health Surveys; 2003.

5. Vallim AL, Osis MJ, Cecatti JG, Baciuk EP, Silveira C, Cavalcante SR. Water exercises and quality of life during pregnancy. Reprod Health 2011;8:14.
6. Moyer CA, Ekpo G, Calhoun CL, Greene J, Naik S, Sippola E, et al. Quality of life, optimism/pessimism, and knowledge and attitudes toward HIV screening among pregnant women in Ghana. Womens Health Issues 2008;18:301-309.

7. Wang P, Liou SR, Cheng CY. Prediction of maternal quality of life on preterm birth and low birthweight: a longitudinal study. BMC Pregnancy Childbirth 2013;13:124.

8. Lau Y. The effect of maternal stress and health-related quality of life on birth outcomes among Macao Chinese pregnant women. J Perinat Neonatal Nurs 2013;27:14-24.

9. Woods SM, Melville JL, Guo Y, Fan MY, Gavin A. Psychosocial stress during pregnancy. Am J Obstet Gynecol 2010;202: 61.e1-e7.

10. Maxson P, Miranda ML. Pregnancy intention, demographic differences, and psychosocial health. J Womens Health (Larchmt) 2011;20:12151223.

11. Senturk V, Abas M, Berksun O, Stewart R. Social support and antenatal depression in extended and nuclear family environments in Turkey: a cross-sectional survey. BMC Psychiatry 2011;11:48.

12. Kingston D, Heaman M, Fell D, Dzakpasu S, Chalmers B. Factors associated with perceived stress and stressful life events in pregnant women: findings from the Canadian Maternity Experiences Survey. Matern Child Health J 2012;16:158-168.

13. Lau Y, Yin L. Maternal, obstetric variables, perceived stress and healthrelated quality of life among pregnant women in Macao, China. Midwifery 2011;27:668-673.

14. Shishehgar S, Dolatian M, Majd HA, Bakhtiary M. Perceived pregnancy stress and quality of life amongst Iranian women. Global J Health Sci 2014;6:270-277.

15. Vollebregt KC, van der Wal MF, Wolf H, Vrijkotte TG, Boer K, Bonsel GJ. Is psychosocial stress in first ongoing pregnancies associated with pre-eclampsia and gestational hypertension? BJOG 2008;115 607-615.

16. Ahmed AE, Albalawi AN, Alshehri AA, AlBlaihed RM, Alsalamah MA. Stress and its predictors in pregnant women: a study in Saudi Arabia. Psychol Res Behav Manag 2017;10:97-102.

17. Li J, Mao J, Du Y, Morris JL, Gong G, Xiong X. Health-related quality of life among pregnant women with and without depression in Hubei, China. Matern Child Health J 2012;16:1355-1363.

18. Seo EJ, Ahn JA, Hayman LL, Kim CJ. The association between perceived stress and quality of life in university students: the parallel mediating role of depressive symptoms and health-promoting behaviors. Asian Nurs Res (Korean Soc Nurs Sci) 2018;12:190-196.

19. Okun ML, Kline CE, Roberts JM, Wettlaufer B, Glover K, Hall M. Prevalence of sleep deficiency in early gestation and its associations with stress and depressive symptoms. J Womens Health (Larchmt) 2013;22:10281037.

20. Hung HM, Tsai PS, Ko SH, Chen CH. Patterns and predictors of sleep quality in Taiwanese pregnant women. MCN Am J Matern Child Nurs 2013;38:95-101.

21. Lagadec N, Steinecker M, Kapassi A, Magnier AM, Chastang J, Robert $\mathrm{S}$, et al. Factors influencing the quality of life of pregnant women: a systematic review. BMC Pregnancy Childbirth 2018;18: 455.

22. Effati-Daryani F, Mirghafourvand M, Mohammad-Alizadeh-Charandabi S, Shiri-Sarand F, Zarei S. Sleep quality and its relationship with quality of life in Iranian pregnant women. Int J Nurs Pract 2017;23.

23. Tsai SY, Lee PL, Lin JW, Lee CN. Cross-sectional and longitudinal associations between sleep and health-related quality of life in pregnant women: a prospective observational study. Int J Nurs Stud 2016;56:45-53.

24. Pires R, Canavarro MC. Examining the links between perceived impact of pregnancy, depressive symptoms, and quality of life during adolescent pregnancy: the buffering role of social support. Matern Child Health J 2014;18:789-800.

25. Shishehgar S, Dolatian M, Majd HA, Bakhtiary M. Perceived pregnancy stress and quality of life amongst Iranian women. Glob J Health Sci 2014;6:270-277.

26. Cohen S, Kamarck T, Mermelstein R. A global measure of perceived stress. 
J Health Soc Behav 1983;24:385-396

27. Lee S, Crockett MS. Effect of assertiveness training on levels of stress and assertiveness experienced by nurses in Taiwan, Republic of China. Issues Ment Health Nurs 1994;15:419-432.

28. Leung DY, Lam T, Chan SS. Three versions of Perceived Stress Scale: validation in a sample of Chinese cardiac patients who smoke. BMC Public Health 2010;10:513.

29. Cox JL, Holden JM, Sagovsky R. Detection of postnatal depression. Development of the 10-item Edinburgh Postnatal Depression Scale. Br J Psychiatry 1987;150:782-786.

30. Lee DT, Yip SK, Chiu HF, Leung TY, Chan KP, Chau IO, et al. Detecting postnatal depression in Chinese women. Validation of the Chinese version of the Edinburgh Postnatal Depression Scale. Br J Psychiatry 1998; $173: 271$

31. Cox J, Holden J. Perinatal Mental Health: A Guide to the Edinburgh Postnatal Depression Scale (EPDS). London, UK: Royal College of Psychiatrists; 2003.

32. Liu X, Tang M. Reliability and validity of the Pittsburgh sleep quality in- dex. Chin J Psychiatry 1996;9:103-107.

33. Ware JE, Kosinski MA, Keller SD. SF-12: How to Score the SF-12 Physical and Mental Health Summary Scales. Boston, MA: The Health Institute, New England Medical Center; 2002.

34. Lam CL, Tse EY, Gandek B. Is the standard SF-12 Health Survey valid and equivalent for a Chinese population? Qual Life Res 2005;14:539-547.

35. Hayes AF. Introduction to mediation, moderation, and conditional process analysis: a regression-based approach. J Educ Meas 2014;51:335337.

36. Lancaster CA, Gold KJ, Flynn HA, Yoo H, Marcus SM, Davis MM. Risk factors for depressive symptoms during pregnancy: a systematic review. Am J Obstet Gynecol 2010;202:5-14.

37. Chang MW, Brown R, Nitzke S, Smith B, Eghtedary K. Stress, Sleep, Depression and dietary intakes among low-income overweight and obese pregnant women. Matern Child Health J 2015;19:1047-1059.

38. Ying L, Yin L. Maternal, obstetric variables, perceived stress and healthrelated quality of life among pregnant women in Macao, China. Midwifery 2011;27:668-673. 